\title{
Analysis of the Problem of Environmental Pollution in Oil Fields, as Well as Ways of Utilization of Oil Production Waste
}

\author{
Vitaly Zhironkin ${ }^{1 *}$, and Michal Cehlar ${ }^{2}$ \\ ${ }^{1}$ National Research Tomsk Polytechnic University, 634050, 30 Lenina st., Tomsk, Russia \\ ${ }^{2}$ Technical University in Kosice, Institute of Earth Resources, Faculty of Mining, Ecology, Process \\ Control and Geotechnologies, Park Komenského 19, 042000 Košice, Slovakia
}

\begin{abstract}
The problems of the current ecological situation in the oil-producing regions are being investigated. The analysis of the impact of the growth of hydrocarbon production on the environment has been carried out. The main causes and sources of pollution have been identified, starting with the process of developing oil and gas fields and including directly extracting and processing oil and gas resources. It is noted that the main reason for the growing environmental hazard is the deterioration and depreciation of equipment and the low share of innovative activity of oil companies. The main methods of utilization of oil production wastes, which are currently used in world practice, have been analyzed. Some technologies for processing oil sludge with obtaining a secondary product are considered. Their main advantages and disadvantages associated with the features of the technological process are described. The possibility of obtaining inert soil, building material, expanded clay and other materials using drill cuttings has been evaluated. The complex separate processing of drilling waste has been determined as the most effective and promising. Its main advantages associated with the complete utilization of all components of oil sludge, as well as the problems of introducing this technology, are given.
\end{abstract}

\section{Introduction}

Due to the insufficient development of the subsoil use strategy, the extensive development of industry, the integration of the necessity and possibilities of scientific regulation of anthropogenic loads, the increasingly threatening degradation of the natural environment in Russia continues. Over the course of many decades, an inefficient, costly economy with a highly deformed structure and deep macroeconomic imbalances has developed in Russia. Russia has turned into a raw material appendage of more developed countries, and in a number of its regions, an extremely dangerous ecological situation has been created, leading to serious consequences for human health.

The current stage of the oil production technology development is characterized by the formation of a significant volume of waste during the development of oil fields. Most of them are currently located in the sludge collectors of barns and are not always subject to further

\footnotetext{
* Corresponding author: v.zhironkin@inbox.ru
} 
utilization [1]. It is this fact that indicates the existence of a number of environmental problems associated with the operation of wells. The greatest danger to the natural environment is presented by production and technological drilling waste, which is accumulated and stored directly on the territory of the drilling site. In their composition, they contain a wide range of pollutants of mineral and organic nature, represented by materials and chemicals [2]. For $1 \mathrm{~m} 3$ of waste, there are up to $68 \mathrm{~kg}$ of polluting organic matter, not counting oil and oil products and mineral pollutants. The percentage ratio between the components of the cuttings can be very diverse depending on the geological conditions, the technical condition of the equipment, and the culture of production [3]. The problem of liquidation of granary oil sludge is currently not fully resolved. This problem is of particular relevance within the framework of preserving the ecological safety of the region in which the extraction takes place. The aim of the work is to determine the most optimal method for the disposal of oil production waste with the receipt of a secondary product. To substantiate the efficiency of separate processing of oil sludge, depending on the conditions of formation and the depth of their occurrence in the sludge ponds.

\section{Materials and Methods}

In world practice, the following methods of processing oil waste are currently used:

- Thermal method: burning in open barns and ovens; drying; pyrolysis; thermal desorption; electric fire treatment.

- Chemical method: hardening by dispersion with hydrophobic reagents based on quicklime or other materials.

- Biological method: biodegradation by introducing (mixing) oily waste into the arable layer of the earth; biodegradation using special bacterial strains, biogenic additives and air supply.

- Physical method: gravitational settling; separation in a centrifugal field; separation by filtration; extraction.

- Physicochemical method: the use of specially selected surfactants (demulsifies, dispersants, wetting agents, etc.), auxiliary substances that affect the change in the state and colloidaldispersed structure in suspended particles in the extra-oil and water phases.

Industrial drilling waste accumulates in dumps at the drilling site and requires subsequent evacuation and placement in a specially designated area for a sludge storage facility (Fig. 1). In this case, the issues of assessing the polluting capacity of waste are of particular importance [4]. The amount and toxicity of pollutants in groundwater depend on this. The main and most potent contaminant during drilling operations is drilling mud. For its preparation, up to two dozen chemical reagents are used, many of which do not have maximum permissible concentrations (MPC) established by the Ministry of Health. The construction of wells and the accompanying accumulation of drilling waste differs from other types of production in the dispersion of drilling facilities, inhomogeneity of waste characteristics and their change over time. 


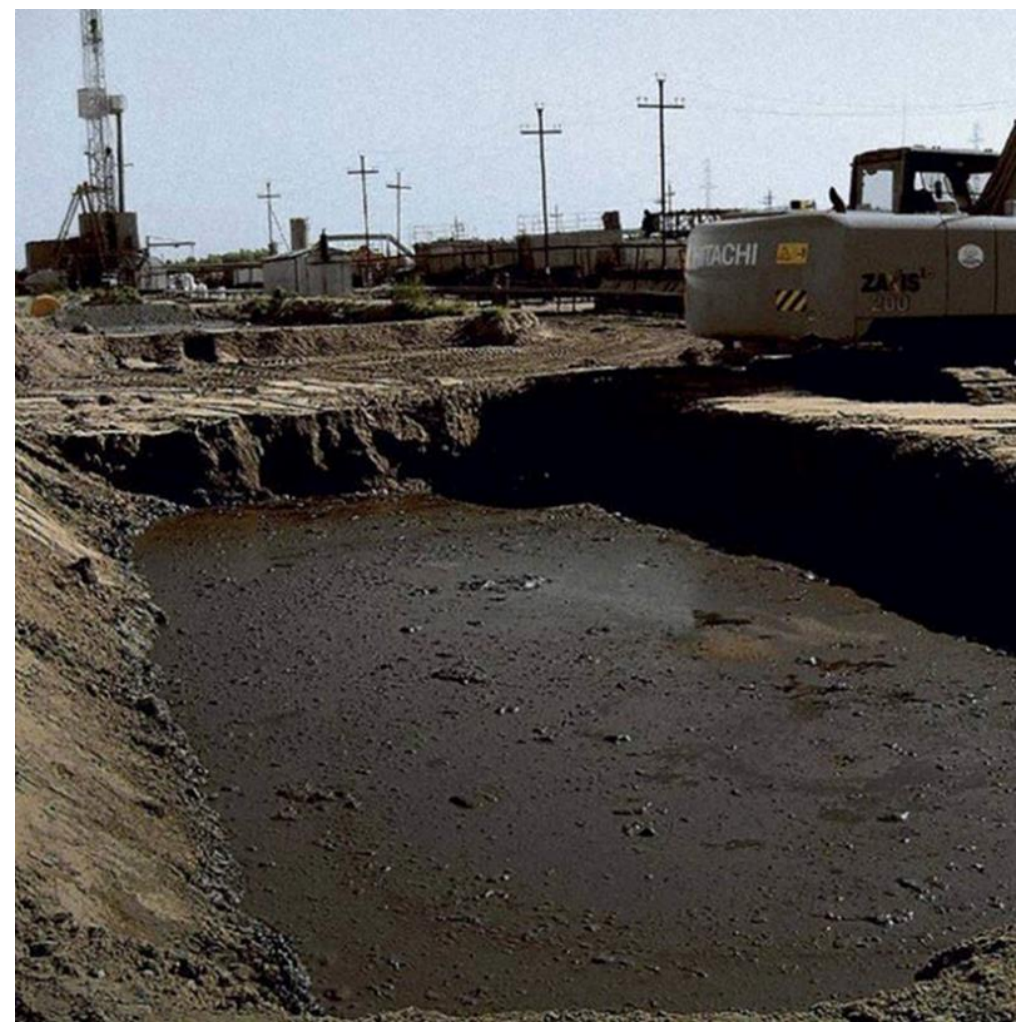

Fig. 1. Waste pit on the drill site.

When performing drilling operations and operating wells, the quality and composition of the used blowout equipment is essential. These measures were aimed at bringing the domestic blowout preventive equipment closer to the level of the best world standards, for example, API16A standard of the American Petroleum Institute. During the exploitation of deposits, huge earth masses are disturbed. Obsolete equipment and methods of economic activity increase the environmental hazard. The transition to new technological methods gives rise to new problems. For example, environmental pollution is observed during well workover. At the same time, hundreds of trips of downhole equipment are carried out. The well is preliminarily pumped with service water until complete degassing. However, in practice, this flushing does not completely remove the film of oil products from the inner and outer surfaces of pipes and equipment. As a result, when the latter is raised to the working site, the well fluid flowing from them contaminates the site and creates a fire hazardous situation in the space around the wellhead. This increases the amount of liquid aggressive waste requiring disposal. In fact, at all the old fields, for decades, the produced formation and wash water was thrown into the evaporation fields. At the old Russian fields of the non-gas producing areas Kulsaryneft and Prorvaneft, the area of mirror gauges is thousands of square kilometers. Huge fields of evaporation of saline water, contaminated with oil products, undoubtedly, negatively affect the biosphere processes in this region. However, if we use the technology for the complete utilization of industrial waters, then the bare bottom of the evaporation fields will become a source of the spread of dry sulphate salts, which will be lifted by the wind and carried over great distances, as is the case in the Aral Sea.

Since oil is the main source of energy and raw material for the production of various fuels and petrochemical products, it is not localized in the producing regions, but intensively spreads to all parts of the Earth, being lost in large quantities during transportation. Therefore, tankers delivering oil products lose about $0.4 \%$ of their cargo (approximately 20.5 thousand tons per 
year). Transit tankers transport about 450 million tons of oil per year. Losses during transportation are at least 11.25 thousand tons. With bilge waters, about 410 tons of oil products are annually supplied to the ocean. Covering the surface with a thin film, oil and oil products disrupt the normal gas exchange, having a negative impact on natural bio- and hydrochemical processes. The ion exchange capacity of soil colloids is impaired, communities of microorganisms are destroyed, and fertility is sharply reduced. Oil has a particularly strong negative effect when it hits the water surface due to its ability to spread with the formation of a thin film. When the concentration of oil pollution is more than $800 \mathrm{mg} / \mathrm{m} 3$, the vital activity of phytoplankton is suppressed, the production of oxygen by algae is reduced. Even in small quantities, oil disrupts the functioning of the nervous system of living organisms, their enzymatic apparatus, causes pathological changes in various tissues of animals, reduces the growth and normal development of algae, and causes their death. In the Caspian Sea, sea waters are polluted, as a result of which the number of commercial fish and seals is decreasing.

It should be noted that drill cuttings, which are waste drilling mud and cuttings, accumulate in sludge pits. Over time, the contents of the barns change, which is associated with the gradual separation of oil sludge into three layers under the action of gravitational forces. On average, it is accepted that oil sludge contains $20-25 \%$ of heavy oil products, $65-75 \%$ of water and $5-10 \%$ of mechanical impurities. With storage and settling, the water content in the sludge decreases significantly, but a significant part of it remains "inside" the oil sludge in a finely dispersed form, being distributed relatively evenly in the mass of oil products (most often related to higher paraffins). During long-term storage of sludge, physicochemical processes characteristic of dispersed systems lead to their separation into layers:

- oil emulsion layer (content of oil products up to $60-80 \%$ );

- a layer of water with an insignificant concentration of oil products (up to 10-15\%) and mechanical impurities;

- bottom layer with a high concentration of mechanical impurities (up to 70-75\%).

It is expedient to process each layer separately using a specific technology and obtain the target product. Some possible ways of utilization of waste generated during the development of oil fields are below.

\section{Results}

Chemical methods are one of the most common methods for processing oil-containing waste. They ensure the receipt of marketable products from waste, for example. building materials. From this point of view, one of the most effective technologies is reagent encapsulation [5]. It consists in the physical and mechanical transformation of the waste into a material neutral for the environment, each particle of which is covered with a hydrophobic shell of calcium carbonate, which is formed when lime is slaked in the presence of water and carbon dioxide. In this case, excess moisture evaporates and waste is rendered harmless. Scientists Yu.V. Logunova, Yu.M.Gerzhberg proposed a prototype of this installation, consisting of a mechanical sludge classifier, a reactor-mixer SSH-2V-700, tanks and storage bins for sludge, water and reagent, dispensers and devices for loading and unloading products. At the Institute of Environmental Safety, Kursk, a mechanized mini-plant for processing oil and oil waste and oil sludge "EKO-5" was developed. In the West, reagent encapsulation technology is known as DKR technology. Developers of Föst-Alpine GmbH have created a mobile unit for the processing of oily waste with a capacity of up to 20 tons per hour. The resulting product is used as a building material for filling roads: GeoGranulate according to TU 5711-004-81436713-2011, recultivator - GeoRecultivant according to TU 2189-006- 81436713-2011, fertilizer for acidic soils GeoCalcite according to TU 2189-002-81436713-2011 [2].

There is a patent in Pure Technologies LLC for a chemical method of thermal extinguishing of oily waste with unslaked lime "Method for processing oily sludge and a device for its 
implementation". This method has a number of advantages: low cost and availability of basic reagents; ease of use of the technological process of the equipment used; the ability to quickly mount and dismantle the equipment used; low energy consumption, confirmed by the results of sanitary and epidemiological studies, a conclusion on the reduction of the hazard class of oil sludge. Despite the practical use of waste (obtaining a powdery product with water-repellent properties) using the described technology, it is not widespread. First, this is due to the need for preliminary preparation of raw materials (waste) according to certain parameters: moisture, particle size distribution and other physical and mechanical properties. Moreover, it is necessary to provide appropriate conditions for the process of its thorough mixing in the mixer. All this requires improved technology and equipment.

Scientists from Russia have developed a new technology for the disposal of oil sludge waste, which ensures the elimination of environmental pollution to a large extent while reducing energy consumption. Its essence lies in the separation of the water-oil emulsion system formed by adding heated water to the original oil sludge, by separation methods, gravity sediment or centrifugation. In the case of a stable water-emulsion system, a method of demulsification of water-organic systems was developed and tested under industrial conditions, based on the effect of a sharp intensification of mass transfer processes under conditions of phase inversion [4]. The advantage of this technology is that the separated oil can be used as a target commercial product with a certain market value. Moreover, the drained water in the technological cycle of oil sludge processing without any additional treatment is returned to the oil sludge storage ponds. It is promising as applied to water-oil emulsions due to the high efficiency of their separation at low energy losses. At the same time, this method does not allow to comprehensively solve the problem of utilization of waste generated during oil production. For which it is necessary to apply other methods in order to process the solid residue. In the course of research on the use of oil production wastes, expanded clay (porous aggregate) based on clay and a solid oil-containing product of oil sludge separation - cake was obtained empirically. The latter is a finely dispersed powder of a dark brown color and is used as a burn-out and swelling additive, as well as to reduce the firing temperature of expanded clay [6]. Studies have shown that mullite is formed in expanded clay - one of the minerals often found in fired ceramic materials. It is of particular value due to its high refractoriness, density, chemical resistance and mechanical strength.

By using cake when obtaining expanded clay, it is possible to achieve a reduction in the cost of building materials, moreover, to preserve and rationally use the available natural raw materials and expand the raw material base for obtaining building materials. The experiments carried out show that the most optimal is the introduction of up to $30 \%$ of cake in the original mixture. In this case, the physical and mechanical properties of the resulting material are improved. Despite the promising nature of this technology and its efficiency in application, it requires preliminary preparation of drill cuttings before using it and the associated additional costs.

There is a method of complex disposal and disposal of drilling waste, in which the decomposition of the oil-water emulsion occurs with the introduction of a coagulant and an oxidizing agent. Powdered iron vitriol $(\mathrm{FeSO} 4 \cdot 7 \mathrm{H} 2 \mathrm{O})$ and bleach $-\mathrm{Ca}(\mathrm{OCl}) 2$ are used as the latter. The separated sediment is mixed with dry sludge and fired to obtain a material for the manufacture of building products. The method is simple, does not require complex equipment and expensive reagents, which simplifies its implementation. In this case, oil products burn out with the formation of heat used for heating, and the receipt of slag used in construction [7]. The proposed method can be considered promising, since the content of oil products after processing decreases approximately from 1500 to 2000 times. However, after preliminary tests, the MPC values for petroleum products (MPC (fishery waters) $=0.3 \mathrm{mg} / 1$ ) were not achieved, which requires further improvement. The disadvantage of this method is the likelihood of the formation of hazardous compounds during the combustion of cuttings, which may contain harmful components, depending on the composition of the drilling fluid. In addition, petroleum products can be used as a commercial product, and in accordance with this technology, they are burned. 
A promising direction is an integrated system for handling drilling waste using them as soil-like recultivation materials for the restoration of a damaged geoenvironment.

For the preparation of oil sludge, geo-container dewatering is proposed, including both dewatering of drilling waste and improving their geo-mechanical properties. Stay of the sludge in self-compaction with the acquisition of strength characteristics. If it is necessary to further enhance the strength properties of dewatered drilling waste (cuttings), it is stacked compounding. In addition, for these purposes, solid low-toxic waste can be used: ash and slag from thermal power plants, fine construction waste, waste from cement production, etc. [8]. Partially dewatered drill cuttings are mixed with bio structuring, immobilizing and hardening additives and formed into a pile or formation. At the same time, along with the improvement of the elasticdeformation properties, the toxicity of the material decreases. The advantage of the method directly related to utilization is the targeted use of reclamation materials as fillers for excavations, relief leveling agents, foundation hardeners, barrier formers and substitutes for soil [9]. Moreover, it can be sold on the territory of sludge pits. The disadvantages of this technology include the inability to extract oil from drill cuttings during preliminary preparation before dehydration, the need to use additional additives, as well as careful control of the compliance of the characteristics of the resulting material in accordance with the intended use.

\section{Conclusion}

Analysis of modern technologies for the utilization of oil sludge makes it possible to determine rational directions for the development of new technological approaches to solving this problem. Due to the fact that the composition of oil sludge is significantly heterogeneous in the depth of receiving tanks and storage ponds, it seems most expedient to use the technology of separate processing of oil sludge using complex disposal methods with a predominance of chemical methods. This approach to the problem allows solving both environmental problems and the problem of rational use of oil products contained in oil sludge and solid phases remaining after separation. As you can see, most of the methods do not allow solving all these problems at once. Each specific method is aimed at one aspect of the issue of drilling waste processing: either the separation of the water-oil emulsion, most often by physical and physicochemical methods, or the disposal of the solid residue mainly by chemical methods. Within the framework of environmental safety, it is relevant and important to combine the most promising and optimal technologies into a system in order to reduce the environmental load of the oil and gas complex on the environment.

\section{References}

1. Yu. G. Bezrodny, Oil Industry Journal, 5, 14 (2019)

2. S. Jacobsen, H. Karianne, G. Ove, O. Tuntland, Overview of Measures Specifically Designed to Prevent Oil Pollution in the Arctic Marine Environment from Offshore Petroleum Activities (Arctic Technology Conference, Labrador, 2016)

3. T. P. Kosulina, D.G. Antoniadi, T.A. Litvinova, O.S. Tsokur, Oil Industry Journal, 11, 149 (2017)

4. C. I. Ossai, U. I. Duru, I. A. Ossai, I. M. Arubi, An Appraisal of Soil Pollution in Oil and Gas Production Environment: A Case Study of Heavy Metals Concentration in Ebocha and Akri Oil Fields (International Oil and Gas Conference and Exhibition in China, Beijing, 2010)

5. T. A. Litvinova, T. V. Vinnikov, T. P. Kosulina, Ecology and Industry of Russia, 5, 112 (2009) 
6. V. A. Kulikov, V. Z. Abdrakhimov, I. V. Kovkov, Bashkir Chemistry journal, 17(3), 81 (2010)

7. V. G. Petrov, E. A. Haraldina, M. A. Shumilova, Bulletin of Udmurt University. Physics. Chemistry, 2, 77 (2011)

8. K. L. Chertes, D. E. Bulls, O. V. Tupitsyna, O. A. Samarin, N. A. Uvarov, E. P. Istomin, A. M. Shterenberg, Ecology and Industry of Russia, 3, 36 (2010)

9. N. A. Safonova, K. L. Chertes, O. V. Tupitsyna, V. N. Pystina, K. D. Kalinkina, V. A. Burlaka, D. E. Bykov, Oil and gas business, 4, 274 (2012) 